\title{
Oral status and Candida colonization in patients with Sjögren's Syndrome
}

\author{
Sertan Ergun $^{1}$, Ali Çekici ${ }^{2}$, Nursen Topcuoglu ${ }^{3}$, Dante-Antonio Migliari ${ }^{4}$, Güven Külekçi ${ }^{3}$, Hakkı Tanyeri ${ }^{1}$, \\ Gülden Isık ${ }^{2}$
}

\author{
${ }^{1}$ Istanbul University, Faculty of Dentistry, Department of Oral Medicine and Surgery \\ ${ }^{2}$ Istanbul University, Faculty of Dentistry, Department of Periodontology \\ ${ }^{3}$ Istanbul University, Faculty of Dentistry, Department of Microbiology \\ ${ }^{4}$ University of Sao Paulo, School of Dentistry, Department of Oral Diagnosis
}

Correspondence:

Istanbul University,

Faculty of Dentistry

Department of Oral Medicine and Surgery

34393 Capa, Istanbul, Turkey

sertanergun@gmail.com

Received: $25 / 05 / 2009$

Accepted: 23/12/2009
Ergun S, Çekici A, Topcuoglu N, Migliari DA, Külekçi G, Tanyeri H, Isık G. Oral status and Candida colonization in patients with Sjögren's Syndrome. Med Oral Patol Oral Cir Bucal. 2010 Mar 1;15 (2):e310-5. http://www.medicinaoral.com/medoralfree01/v15i2/medoralv15i2p310.pdf

Article Number: 2923 http://www.medicinaoral.com/

(C) Medicina Oral S. L. C.I.F. B 96689336 - pISSN 1698-4447 - eISSN: 1698-6946

eMail: medicina@medicinaoral.com

Indexed in:

-SCI EXPANDED

-JOURNAL CITATION REPORTS

-Index Medicus / MEDLINE / PubMed

-EMBASE, Excerpta Medica

-SCOPUS

-Indice Médico Español

\begin{abstract}
Objective: To determine the oral status, salivary flow rate, Candida carriage in saliva, and prevalence of Candida albicans colonization in several areas of the mouth in patients with primary and secondary Sjögren's syndrome as opposed to those of healthy subjects.

Study design: Thirty-seven patients with Sjögren's syndrome (SS), [14 patients with primary SS (SS-1) and 23 patients with secondary SS (SS-2)], along with 37 healthy controls were examined in regard to number of teeth, pro-bing pocket depth (PPD), approximal plaque index (API), bleeding on probing (BOP), presence of prosthetic appliances and smoking habits. Salivary flow rate (SFR), Candida carriage in saliva, presence of Candida albicans colonization on buccal, angular, palatal and sulcular areas, on dentures and on the tongue's dorsal surface were determined. Statistical analyses were performed using the 2-tailed Fisher exact and Kruskal-Wallis test.

Results: No statistically significant difference was found between SS-1 and SS-2 groups based on the parameters analysed. Statistically significant differences were observed between patients with SS and healthy subjects in terms of SFR, oral signs and symptoms, API, BOP, C. albicans colonization on tongue and buccal area, and Candida carriage in saliva. In the gingival crevicular fluid positive C. albicans colonization was found in only one subject of SS subgroup.

Conclusions: SS patients carry a higher risk of having periodontitis and are more predisposed to develop candidiasis. C. albicans is scarcely detected in gingival crevicular fluid despite high scores on C. albicans colonization in different areas of the oral cavity in SS patients.
\end{abstract}

Key words: Sjögren's syndrome, candida colonization, oral and periodontal status. 


\section{Introduction}

Sjögren's syndrome (SS) is a systemic chronic autoimmune disease characterized by severe dryness of the mouth (xerostomia) and eyes (keratoconjunctivitis sicca), along with major salivary gland enlargement caused by lymphoid infiltration (1). SS is classified as secondary (SS-2) when it is associated with other autoimmune diseases such as rheumatoid arthritis (RA) and systemic lupus erythematosus (SLE), and as primary (SS-1) when there is no other connective tissue disease $(1,2)$. Several epidemiologic studies on SS have shown that the disease affects primarily females (nine fold higher than men) during the fourth and fifth decades of life (3).

Subjective xerostomia has been reported in higher percentages $(75.18 \%$ to $91.84 \%$ ) in patients with SS (4). As a consequence, these patients are prone to develop a variety of signs and symtoms such as dental caries, angular chelitis, redness of the tongue, atrophy of filiform papilae, halitosis, difficulties and pain on swallowing and burning syndrome (4). Studies have demonstrated a higher gingival bleeding and plaque index in subjects with hyposalivation but without showing a correlation between salivary flow rate and gingival bleeding index or plaque index resulting from higher risk of having periodontitis $(5,6)$.

A continuous flow of saliva is important to prevent oral colonization by Candida. Various investigators have reported a high prevalence of oral Candida species in patients with SS as compared with those of healthy controls $(5,7-11)$, while others have found that there is no significant difference between patients with SS and healthy controls in terms of presence of candidiasis $(8$, 12). Most reports indicate that C. albicans is the predominant yeast isolated in gingival crevicular fluid and in periodontal pockets of the periodontal patients as well as in healthy subjects, although Candida glabrata and Candida tropicalis have also been found, albeit infrequently $(9,11,13)$.

The aim of this study was to compare the oral status, oral hygiene, smoking habits, salivary flow rate, presence of prosthetic appliances, number of teeth, bleeding on probing, approximal plaque index, probing pocket depth, Candida carriage in saliva, and prevalence of C. albicans colonization in gingival crevicular fluid, on dentures, on buccal, angular and palatal areas, and on the tongue's dorsal surface of SS-1 and SS-2 patients as opposed to those of healthy subjects.

\section{Study design}

Subject population consisted of 37 patients with SS, of whom 14 patients were diagnosed as having primary SS (SS-1) and 23 patients with secondary SS (SS-2), and 37 healthy individuals. SS patients were diagnosed at Istanbul University, Faculty of Medicine, Department of Rheumatology, based on the recently modified interna- tionally agreed-on criteria for Sjögren's syndrome. Oral and periodontal examination and mycological analyses were performed in all groups. Selection criteria included only subjects who did not receive periodontal, antibiotic or antimycotic therapy over the last three months. The study was approved of the Ethic Committee of Istanbul University, Faculty of Medicine.

Oral examination comprised the evaluation the clinical aspects of the lips, the tongue, the floor of the mouth, the gingiva, the cheeks, and the palate, focusing mainly on the signs and symptoms related to SS. Presence of any systemic disease as well as smoking habits were recorded. All oral clinical examinations were performed by the same examiner from the Department of Oral Medicine and Surgery.

Periodontal examination included number of teeth (NT), bleeding on probing (BOP) (expressed as the percentage of sites that bled upon gentle probing), approximal plaque index (API) (expressed as the percentage of sites which presented plaque), probing pocket depth (PPD) (the distance from the crest of the gingival margin to the base of the pocket). BOP, API and PPD were measured at 4 sites of each tooth: buccal, mesial, lingual and distal. All the measurements were performed by the same examiner attendant at the Department of Periodontology using a $0.5 \mathrm{~mm}$-diameter Hu-Friedy periodontal probe with Williams's markings.

Mycological examinations included Candida carriage in saliva and prevalence of $C$. albicans colonization on dentures, in gingival crevicular fluid, on the tongue, and on buccal, angular and palatal areas. Samples of buccal, angular, palatal areas, tongue and denture surfaces were taken by a sterile cotton swab, and subgingival plaque samples were taken by sterile paper points. All samples were transferred into a vessel with $1 \mathrm{ml}$ saline solution and mixed 20 seconds for homogenization by a Vortex mixer. Aliquots of $0.01 \mathrm{ml}$ of swab, subgingival plaque and saliva samples were plated onto Sabouroud Agar (Merck, Darmstad, Germany) and incubated at $37^{\circ} \mathrm{C}$ and $45^{\circ} \mathrm{C}$ for $48 \mathrm{~h}$. The numbers of the typical colonies of the saliva samples were enumerated and calculated as $\mathrm{CFU} / \mathrm{ml}$. C. albicans isolates were identified by the germ tube test.

Whole unstimulated saliva was collected in a sterilized plastic tube for 5 minutes. The salivary flow rate (SFR) was recorded as $\mathrm{mL} / \mathrm{min}$. Subjects with SS were also divided into two groups: one with a non-pathological level of resting saliva (normal saliva group $>0.1 \mathrm{~mL} / \mathrm{min}$ ) and the other with pathological level (low saliva group: $<0.1$ $\mathrm{mL} / \mathrm{min}$ ). Statistical analyses were performed by using the Chi-Square and Kruskall Wallis one-way ANOVA tests. Statistical significance was set at $\mathrm{p}<0.05$.

\section{Results}

The age of the patients with SS ranged from 26 to 78 years (mean, 53.27), while the age of the healthy sub- 
jects ranged from 25 to 94 years (mean, 54.27). There was no statistical significant difference between the groups in terms of age, gender and smoking habits ( $p$ $>0.05$ ). Rheumatoid arthritis was the most commonly seen autoimmune disease in secondary SS group (SS-2) observed in 21 (91.30\%) patients. Systemic lupus erythmatosus was found in only two patients $(8.70 \%)$.

Oral examination of the patients with SS revealed no statistically significant difference between SS-1 and $\mathrm{SS}-2$ patients in regard to the oral signs evaluated ( $\mathrm{p}$ $>0.05$ ). Significant differences were observed between patients with SS and healthy subjects in terms of the clinical oral findings associated with SS (Table 1).

There were 10 edentulous patients with SS (three with SS-1 and seven with SS-2) and they all were using total prosthesis, while 12 healthy subjects were using total prosthesis (p $>0.05$ ). Dentate patients with SS (11 with SS-1 and 16 with SS-2) had $12.70 \pm 1.75$ teeth, while dentate healthy subjects had $12.73 \pm 1.89$ teeth $(p>0.05)$. There was no statistically significant difference between patients with SS and healthy controls regarding to their tooth brushing habits $(\mathrm{p}>0.05)$. There was no statistically significant difference between patients with SS-1 and SS-2 regarding their periodontal status $(p>0.05)$; however, as shown in the table 2 , a statistically significant difference was observed between patients with SS and healthy subjects in regard to BOP $(p=0.025)$ and API $(p=0.001)$.
The difference between the SS patients and the healthy subjects in terms of SFR was statistically significant ( $p$ $=0.001$ ). No statistical significant difference was found between the two subgroups of SS patients regarding their mean ustimulated whole salivary flow rate $(p>$ 0.05).

The Candida counts in saliva was lower than $10^{3} \mathrm{CFU} /$ $\mathrm{mL}$ in five SS-1 (35.71\%), nine SS-2 (39.13\%) and 13 healthy subjects $(86.66 \%)$, between $10^{3}$ and $10^{4} \mathrm{CFU} /$ $\mathrm{mL}$ in four SS-1 $(28.6 \%)$, five SS-2 $(21.74 \%)$ and two healthy subjects $(13.33 \%)$ and higher than $10^{4} \mathrm{CFU} /$ $\mathrm{mL}$ in five SS-1 (35.71\%) and nine SS-2 (39.13\%) subjects. Statistically significant difference was observed between patients with SS and healthy controls in terms of Candida carriage in saliva ( $\mathrm{p}=0.001)$. Despite the considerable differences in the median $\mathrm{CFU} / \mathrm{ml}$ for each subgroup of SS, these differences were not statistically significant $(\mathrm{p}>0.05)$. C. albicans colonization in the gingival crevicular fluid was detected in only one tooth of one SS-1 subject. There was no statistically significant difference between SS-1 and SS-2 subjects on the prevalence of $C$. albicans colonization on dentures, in gingival crevicular fluid, on buccal, angular, palatal areas and the tongue $(p>0.05)$. Statistically significant differences were observed between the patients with SS and healthy controls in terms of $C$. albicans colonization on tongue and buccal area (Table 2).

Table 1. Positive objective and subjective signs on oral clinical examination of the patients with SS

\begin{tabular}{|c|c|c|c|}
\hline & SS & HS & \\
& $(\mathrm{n}=37)$ & $(\mathrm{n}=37)$ & $\mathrm{p}$ \\
& $\mathrm{N}, \%$, & $\mathrm{N}, \%$, & \\
\hline Angular Chelitis & $8(21.62 \%)$ & $0(0 \%)$ & $0.005(\mathrm{~S})$ \\
\hline Oral Ulcerations & $13(35.13 \%)$ & $0(0 \%)$ & $0.0001(\mathrm{~S})$ \\
\hline Atrophic Mucosa & $28(75.67 \%)$ & $3(8.10 \%)$ & $0.0001(\mathrm{~S})$ \\
\hline Dry Mucosa & $23(62.16 \%)$ & $1(2.70 \%)$ & $0.0001(\mathrm{~S})$ \\
\hline Reddened Mucosa & $23(62.16 \%)$ & $5(13.51 \%)$ & $0.0001(\mathrm{~S})$ \\
\hline Atrophy of Filiform & $18(48.65 \%)$ & $4(10.81 \%)$ & $0.001(\mathrm{~S})$ \\
\hline Papilla & $32(86.49 \%)$ & $5(13.51 \%)$ & $0.0001(\mathrm{~S})$ \\
\hline Xerostomia & $29(78.38 \%)$ & $5(13.51 \%)$ & $0.0001(\mathrm{~S})$ \\
\hline Burning Sensation & $23(62.16 \%)$ & $4(10.81 \%)$ & $0.0001(\mathrm{~S})$ \\
\hline Pain on Swallowing & $30(81.08 \%)$ & $3(8.10 \%)$ & $0.0001(\mathrm{~S})$ \\
\hline Dysgeusia & $22(59.46 \%)$ & $0(0 \%)$ & $0.0001(\mathrm{~S})$ \\
\hline Hypersensitivity & & &
\end{tabular}

SS: Sjögren's syndrome

HS: healthy subjects; N: number; \%: percentage; S: statistical significant; NS: no statistical significant 
Table 2. The periodontal status and positive Candida albicans colonization (PCAC) in different areas of the oral cavity of the patients with SS.

\begin{tabular}{|c|c|c|c|}
\hline & $\begin{array}{c}\mathrm{SS} \\
\text { mean } \pm \text { SEM / } \\
\mathrm{N}, \%\end{array}$ & $\begin{array}{c}\text { HS } \\
\text { mean } \pm \text { SEM / } \\
\mathrm{N}, \%,\end{array}$ & $\mathrm{P}$ \\
\hline Number of Teeth (NT) & $17.73 \pm 8.42 / \mathrm{n}=27$ & $24.26 \pm 3.82 / \mathrm{n}=25$ & 0.07 (NS) \\
\hline $\begin{array}{l}\text { Bleeding on Probing } \\
(\mathrm{BOP})(\%)\end{array}$ & $62.29 \pm 30.35 / \mathrm{n}=27$ & $42.96 \pm 29.70 / n=25$ & $0.025(\mathrm{~S})$ \\
\hline $\begin{array}{l}\text { Approximal Plaque } \\
\text { Index } \\
(\mathrm{API})(\%)\end{array}$ & $76.84 \pm 26.22 / \mathrm{n}=27$ & $48.94 \pm 27.54 / \mathrm{n}=25$ & $0.001(\mathrm{~S})$ \\
\hline $\begin{array}{l}\text { Probing Pocket Depth } \\
(\mathrm{PPD})(\mathrm{mm})\end{array}$ & $1.88 \pm 0.41 / \mathrm{n}=27$ & $1.95 \pm 0.63 / \mathrm{n}=25$ & $\begin{array}{l}0.643 \\
(\mathrm{NS})\end{array}$ \\
\hline (PCAC) on tongue & $\begin{array}{c}28 / \mathrm{n}=37 \\
75.68 \%\end{array}$ & $\begin{array}{l}19 / \mathrm{n}=37 \\
51.35 \%\end{array}$ & $0.030(\mathrm{~S})$ \\
\hline $\begin{array}{l}\text { (PCAC) on the buccal } \\
\text { area }\end{array}$ & $\begin{array}{c}21 / \mathrm{n}=37 \\
56.76 \%\end{array}$ & $\begin{array}{c}11 / \mathrm{n}=37 \\
29.73 \%\end{array}$ & $0.019(\mathrm{~S})$ \\
\hline $\begin{array}{l}\text { (PCAC) on the palatal } \\
\text { mucosa }\end{array}$ & $\begin{array}{c}12 / \mathrm{n}=37 \\
32.43 \%\end{array}$ & $\begin{array}{c}13 / \mathrm{n}=37 \\
35.14 \%\end{array}$ & $0.806(\mathrm{NS})$ \\
\hline $\begin{array}{l}\text { (PCAC) on the angular } \\
\text { area }\end{array}$ & $\begin{array}{c}10 / \mathrm{n}=37 \\
27.03 \%\end{array}$ & $\begin{array}{l}4 / n=37 \\
10.81 \%\end{array}$ & 0.075 (NS) \\
\hline $\begin{array}{l}\text { (PCAC) in gingival } \\
\text { crevicular fluid }\end{array}$ & $\begin{array}{l}1 / \mathrm{n}=27 \\
3.70 \%\end{array}$ & $\begin{array}{l}1 / \mathrm{n}=24 \\
4.16 \%\end{array}$ & $0.750(\mathrm{NS})$ \\
\hline (PCAC) on dentures & $16 / n=20 \quad 80.00 \%$ & $\begin{array}{l}12 / \mathrm{n}=22 \\
154.54 \%\end{array}$ & $0.195(\mathrm{NS})$ \\
\hline
\end{tabular}

SS: Sjögren's syndrome HS: healthy subjects; N: number; \%: percentage; S: statistical significant; NS: no statistical significant

\section{Discussion}

For a preventive measure in the oral cavity of subjects with SS, it is important that practitioners have a thorough knowledge about the effect of decreased salivary flow on the oral status. In this study, oral status and $C$. albicans colonization of the patients with SS-1 and SS-2 were compared with those of control healthy subjects. Concerning the periodontal status, a statistically significant difference was found between patients with SS and healthy controls, which is in agreement with findings reported in other similar studies $(5,6,14)$, although there is no consensus in this matter, since other studies showed that SS patients did not present a higher risk for periodontitis compared with that of the general population $(4,7,14-16)$.
Xerostomia and objective oral signs were highly prevalent in SS patients compared with those of the control subjects. But both groups, SS and controls, did not present significant difference in terms of tooth-brushing efficiency. Thus, the higher prevalance of peridontitis in SS patients is very likely to be due to their low SFR. Most of the studies did not find a statistically significant difference between primary and secondary SS patients in terms of their periodontal status $(4,7,14,17)$. Our results are in agreement with these findings. Accordingly, the periodontal status seems not to be affected by the type of SS.

Clinically, C. albicans can be cultured from swabs of the buccal mucosa, tongue, teeth, denture surfaces, and dental plaque samples. The flushing effect of saliva and 
anti-candidal salivary components such as lysozyme, histatins, lactoferrin, and calprotectin are the innate host defenses which act to remove or kill invading yeasts (8). The decreased salivary flow means the decreased host defense. In our study, candidal colonization on the buccal epithelial and the dorsal tongue was found to be in higher in SS patients than in healthy controls. In colonized individuals with no clinical symptoms of candidiasis, C. albicans is most frequently found on the dorsum of the tongue. Although Almståhl \& Wikström (9) did not find an increase of frequency of Candida in subjects with hyposalivation, those authors did not analyse Candida colonization on the tongue's dorsal surface, which is the main ecological niche for Candida in the oral cavity.

Denture wearing is one of the major predisposing factors in humans for oral candidiasis. In denture wearers, the fitting surface of the denture is the main reservoir of the yeasts (10). Angular chelitis is commonly associated with denture-induced stomatitis. In our study, no statistically significant difference was found between SS and healthy subjects on the prevalence of $C$. albicans colonization on dentures, palatal and angular areas who use dentures with similar cleaning habits.

As there are limited findings in healthy subjects, yeasts especially $C$. albicans have been recovered from periodontal pockets of patients with chronic periodontitis in different rates (7.1 to19.6\%). The gingival crevicular fluid (GCF) is considered a transudate, a passage of fluid from bloodstream $(18,19)$. But it is also known that amount of GCF increases with periodontal disease, which leads us to think that GCF renews itself continuously (18). In our study, SS patients and control subjects showed slight to moderate signs of inflammation. Despite high scores of PCAC in different areas of the mouth in SS-1 and SS-2 patients, PCAC in the GCF was found in only one SS subject (2.7\%) of SS-1 subgroup. The very low prevalence of $C$. albicans in the GCF should be related to the continuous flow rate of this area, making the enviroment difficult for fungi colonization. Rhodus and Michalowicz (14) found almost the same result in their pilot study, in which they compared the periodontal status and prevalence of sulcular $C . a l$ bicans between subjects with SS-1 and healthy control subjects.

There were direct correlations between PCAC on buccal area and dry mucosa, hypersensitivity and pain on swallowing with no specific reason. Additionally, a weak correlation between Candida carriage in saliva and pain on swallowing was detected. Logemann et al. reported that xerestomia affects the sensory process of swallowing (20). It is well known that positive Candida carriage in saliva is mostly the result of the lower levels of salivary flow rate. From this available evidence, it can be assumed that difficulties and pain on swallowing might occur due to positive Candida carriage in saliva. But more studies with higher number of patients with $\mathrm{SS}$ are needed to confirm or refuse this association.

The limitation of the present study were the reduced number of the patients with SS, which was mostly due to the newly diagnosed SS patients according to the recently modified internationally agreed-on criteria for Sjögren's Syndrome since 2004 and the lack of determining of the number of decayed, missing and filled teeth which is known as DMF-T index.

\section{Conclusion}

Patients suffering from SS may experience great impairment in their general health. Regarding oral involvement xerostomia is a predominant finding and causes many oral discomforts, including infection by Candida albicans and periodontitis. Oral hygiene should be encouraged for all these patients in addition to palliative measures to alleviate dry mouth.

\section{References}

1. Margaix-Muñoz M, Bagán JV, Poveda R, Jiménez Y, Sarrión G. Sjögren's syndrome of the oral cavity. Review and update. Med Oral Patol Oral Cir Bucal. 2009;14:E325-30.

2. Soto-Rojas AE, Kraus A. The oral side of Sjögren syndrome. Diagnosis and treatment. A review. Arch Med Res. 2002;33:95-106.

3. Alamanos Y, Tsifetaki N, Voulgari PV, Venetsanopoulou AI, Siozos C, Drosos AA. Epidemiology of primary Sjögren's syndrome in north-west Greece, 1982-2003. Rheumatology (Oxford). 2006;45:187-91.

4. Boutsi EA, Paikos S, Dafni UG, Moutsopoulos HM, Skopouli FN. Dental and periodontal status of Sjögren's syndrome. J Clin Periodontol. 2000;27:231-5.

5. Celenligil H, Eratalay K, Kansu E, Ebersole JL. Periodontal status and serum antibody responses to oral microorganisms in Sjögren's syndrome. J Periodontol. 1998;69:571-7.

6. Najera MP, Al-Hashimi I, Plemons JM, Rivera-Hidalgo F, Rees TD, Haghighat N, et al. Prevalence of periodontal disease in patients with Sjögren's syndrome. Oral Surg Oral Med Oral Pathol Oral Radiol Endod. 1997;83:453-7.

7. Tseng CC. Periodontal status of patients with Sjögren's syndrome: a cross-sectional study. J Formos Med Assoc. 1991;90:109-11.

8. Cannon RD, Chaffin WL. Colonization is a crucial factor in oral candidiasis. J Dent Educ. 2001;65:785-7.

9. Almståhl A, Wikström M. Oral microflora in subjects with reduced salivary secretion. J Dent Res. 1999;78:1410-6.

10. Scully C, El-Kabir M, Samaranayake LP. Candida and oral candidosis: a review. Crit Rev Oral Biol Med. 1994;5:125-57.

11. Järvensivu A, Hietanen J, Rautemaa R, Sorsa T, Richardson M. Candida yeasts in chronic periodontitis tissues and subgingival microbial biofilms in vivo. Oral Dis. 2004;10:106-12.

12. Keene HJ, Horton IM, Handler SF. Streptococcus mutans approximal plaque index as a new epidemiologic tool for defining the parameters of Streptococcus mutans infection in human populations. Arch Oral Biol. 1981;26:345-55.

13. Cannon RD, Chaffin WL. Oral colonization by Candida albicans. Crit Rev Oral Biol Med. 1999;10:359-83.

14. Rhodus NL, Michalowicz BS. Periodontal status and sulcular Candida albicans colonization in patients with primary Sjögren's Syndrome. Quintessence Int. 2005;36:228-33.

15. Kuru B, McCullough MJ, Yilmaz S, Porter SR. Clinical and microbiological studies of periodontal disease in Sjögren syndrome patients. J Clin Periodontol. 2002;29:92-102. 
16. Pers JO, D’Arbonneau F, Devauchelle-Pensec V, Saraux A, Pennec YL, Youinou P. Is periodontal disease mediated by salivary BAFF in Sjögren's syndrome? Arthritis Rheum. 2005;52:2411-4.

17. Kindelan SA, Yeoman CM, Douglas CW, Franklin C. A comparison of intraoral Candida carriage in Sjögren's syndrome patients with healthy xerostomic controls. Oral Surg Oral Med Oral Pathol Oral Radiol Endod. 1998;85:162-7.

18. Brill N. The gingival pocket fluid.Studies of its occurrence, composition and effect. Acta Odontol Scand. 1969;2:159.

19. Cimasoni G. Crevicular fluid updated. In myers H (ed): Monographs in Oral Science, vol 12 Basel, S Krager, 1983.

20. Logemann JA, Pauloski BR, Rademaker AW, Lazarus CL, Mittal B, Gaziano J, et al. Xerostomia: 12-month changes in saliva production and its relationship to perception and performance of swallow function, oral intake, and diet after chemoradiation. Head Neck. 2003;25:432-7.

\section{Acknowledgement:}

We would like to thank Assoc. Prof. Dr. Gonca Mumcu, University of Marmara, for her precious help by making of statistical analyses of this study.

Conflict of interest and source of funding statement: The authors declare that they have no conflict of interests. The study was selffunded by the authors and their institution.

This study was established under approval of the Ethic Committee of Istanbul University, Faculty of Medicine. No:682 